\title{
Linx
}

Revue des linguistes de l'université Paris X Nanterre

$59 \mid 2008$

Les conjonctions en diachronie : parcours sémantiques

\section{La locution ainsi que en Moyen Français : questions sur l'évolution des conjonctions}

\section{Annie Bertin}

\section{OpenEdition}

\section{Journals}

Édition électronique

URL : https://journals.openedition.org/linx/625

DOI : $10.4000 /$ linx.625

ISSN : 2118-9692

Éditeur

Presses universitaires de Paris Nanterre

Édition imprimée

Date de publication : 1 décembre 2008

Pagination : 61-82

ISSN : 0246-8743

Référence électronique

Annie Bertin, «La locution ainsi que en Moyen Français : questions sur l'évolution des conjonctions », Linx [En ligne], 59 | 2008, mis en ligne le 01 janvier 2012, consulté le 07 décembre 2022. URL : http:// journals.openedition.org/linx/625; DOI : https://doi.org/10.4000/linx.625 


\title{
La locution ainsi que en Moyen Français : questions sur l'évolution des conjonctions
}

\author{
Annie Bertin \\ Université Paris Ouest Nanterre La Défense - UMR Modyco
}

Retracer l'évolution de la locution ainsi que est un sujet de choix pour l'historien $\mathrm{du}$ français qui doit aborder de multiples questions : l'origine du premier élément ainsi qui reste énigmatique; la concurrence de cette forme apparue vers la fin du $13^{\mathrm{e}}$ siècle et de la forme plus ancienne ainsi com, le rapport entre les formes conjointes ainsi que (/ com) et les formes disjointes correspondantes, la place de ainsi que (/ com) dans le paradigme des locutions formées sur si (si, issi, autresi, aussi que/com) dont tous les termes disparaissent hormis précisément la locution ainsi que et les formes disjointes si...que et aussi ...que avec cependant une spécialisation sémantique nette. En FM la locution ainsi que fait partie de ces «marqueurs d'identité similative » (désormais MIS), pour reprendre la terminologie de Haspelmath \& Buchholz (1998), dont le statut subordonnant ou coordonnant fait question. Pierrard, Hadermann et Van Raemdonck (2006), par la confrontation du fonctionnement des MIS (ainsi, de même, tel, aussi (bien)) et $\mathrm{MIS}_{\text {que }}$ (ainsi que, comme, de même que, tel que, aussi bien que) ont montré que l'opposition subordonnant $v$ s coordonnant, selon les critères définis par Lehmann (1988) pour la subordination, ne peut se décrire comme un continuum «simple»; pour rendre compte de façon systématique de cette diversité, ils concluent à la nécessité de creuser la sémantique de ces termes et leurs différents degrés de grammaticalisation. Cela confirme l'utilité d'observer le fonctionnement de ces marqueurs dans des états antérieurs du français. On ne prétendra pas aborder toutes les questions, précédemment évoquées que pose l'évolution de ainsi que, même si l'on est amené à les croiser, mais cette étude devrait permettre d'éclairer la question du sémantisme de ainsi que 
à partir de l'évolution de ses emplois. On s'attachera à décrire les constructions comportant le marqueur ainsi que en moyen français $\left(14^{\mathrm{e}}-15^{\mathrm{e}}\right.$ siècle, désormais $\left.\mathrm{MF}\right)$, état de la langue bien documenté par un corpus informatisé ${ }^{1}$; dans une première partie, seront considérées les constructions avec subordonnée, dans une deuxième partie, les constructions elliptiques. Dans la troisième et dernière partie, après avoir comparé les emplois du MF aux emplois actuels, on s'interrogera sur deux points : le parcours menant à la restriction sémantique de cette locution et sa relation au mécanisme de la grammaticalisation.

\section{Ainsi que comme introducteur de proposition en Moyen Français}

Dans son étude des systèmes comparatifs à deux termes de l'ancien français, Jonas (1971) utilise prudemment, à propos des syntagmes du type ainsi que, le terme d'articulant. Sa prudence terminologique, même si elle n'est pas explicitée, rejoint les débats récents et anciens sur le statut des mots subordonnants et sur les mécanismes de la subordination. On constate en effet que la locution ainsi que apparait dans divers types de constructions : elle introduit des propositions complètes tout comme des groupes averbaux, et instaure, entre les constituants qu'elle relie, des rapports syntaxiques et sémantiques variés, ce qui fait difficulté pour la catégoriser ${ }^{2}$.

Les relations entre propositions, marquées par ainsi que ressortissent à différents champs sémantiques : temps, comparaison, conséquence, manière, notions qui semblent bien correspondre à des catégories sémantiques translinguistiques, dans lesquelles s'inscrit notre description.

\subsection{La valeur comparative ${ }^{3}$}

L'étiquette "comparaison» recouvre, dans notre corpus, deux types de relations : la similitude entre deux procès (1), et le commentaire méta-énonciatif porté par ainsi que $q$ sur l'énoncé $p(2)$. Par ailleurs, tout en exprimant une identité de manière, ainsi que $q$ peut constituer un complément obligatoire à la rection du verbe de $p$ (3).

1.1.1 La similitude est assez souvent, sans que ce soit systématique, soulignée dans la principale par un adverbe (ainsi, aussi, semblablement) soulignant la corrélation :

\footnotetext{
${ }^{1}$ L'étude s'appuie sur le dépouillement d'un corpus de 1155 occurrences fournies par la Base de Moyen Français (graphies ainsi/einsi que).

${ }^{2}$ On note que le catégoriseur morpho-syntaxique de la base Frantext, dans la partie catégorisée de celle-ci, attribue de ce fait à ainsi que la catégorie $[\mathrm{X}]$.

${ }^{3}$ Bien que la temporalité semble constituer une source privilégiée du développement des conjonctions, au point que l'on a pu en faire un caractère universel de ce phénomène (Kortmann 1997 : 155 ; Lühr 1989), on examinera en premier lieu les comparatives, les plus représentées numériquement en $\mathrm{MF}$, et qui semblent constituer la valeur prototypique de la locution.
} 
1) Ainsi que en chaschune euvre constance est trouvee la mere, ainsi de toute doctrine et discipline negligence est la marastre. Jean Daudin, De la erudition, 1360, p. 28

L'adverbe tout devant la locution ${ }^{4}$ souligne très fréquemment l'exactitude du rapprochement analogique, lequel est marqué ou non dans la principale par un adverbe signalant la corrélation ${ }^{5}$ :

2) Mais tout ainsi que vous m'avez aidié a mon grant besoing en bonne foy, je vous aideray loyaument au vostre. Berinus t. 2, 1350, p.172

3) Et pourtant, à propos dit Saint Bernart que ceste vie mortele puet estre à un chascun figurée à la prison, car tout ainsi que la closture de la prison detient le prisonnier si à destroit que user ne puet de ses mesmes vouloirs n'acomplir ses desirs ains communement à tout le contraire, semblablement l'ame raisonnable, qui la plus noble partie est de l'omme, sans laquelle le corps n'est fors terre et pourreture, est detenue emprisonnée et liée dedens le corps. Christine de Pizan, Epistre de la prison de vie bumaine, 1416, p. 19

4) Car tout ainsi que en esté, Le formy fait sa garnison, Pour vivre toute la saison, Ainsi chascun doit entendre, Retenir et aussi apprendre Aucun bien des sa jeunesse. Charles d'Orléans, le Livre contre tout peche, 1404, p. 549

5) mais aler vous convendra hors du royaume de Blandie, tout ainsi que Berinus mon pere s'en ala, quant il fut chaciez. Berinus t. 2, 1350, p. 168

On observe que cet emploi fait souvent intervenir en $q$ un prédicat « décoloré » qui «représente une reprise du prédicat principal ou un pro-verbe » (Pierrard \& alii 2006 :138) ; à côté de pures répétitions, comme en (2-3-5), q fait également apparaître souvent un substitut verbal ${ }^{6}$ :

6) Si eurent aulcuns parlemens ensamble, ainsi que seigneurs et dames ont. Froissart, Chroniques III, 1390, p. 233

7) si advint un jour que je aloie traiant de mon art, ainsi que enfans font. Berinus t. 2, 1350, p. 12

\footnotetext{
${ }^{4}$ On rencontre une seule occurrence présentant un autre adverbe modifiant ainsi que: "Si devise de la loutre et de toute sa nature. Et portent autant comme furons aucunnes foiz leurs cheaux, plus ou moins einsi que les furons". Gaston Phebus, Livre de Chasse, 1387, p.105. La construction est elliptique mais relève de la comparaison.

${ }^{5}$ L'emploi de tout, est si fréquent que l'on peut se demander s'il est perçu comme un moyen de renforcement sémantique ou comme un constituant lexicalisé ou en voie de lexicalisation de la locution; c'est la seconde interprétation qui ultérieurement se manifeste dans le Dictionnaire de l'Académie (1694), à l'article ainsi que: Tout ainsi que. Il a le mesme sens \& le mesme usage [que ainsi que].

${ }^{6}$ Le fait d'utiliser un substitut verbal autre que le verbe (faire ou un auxiliaire) renforce l'hypothèse qu'il s'agit d'une construction particulière.
} 
8) Il advint un jour que saint Germain s'en ala a Romme, ainsi que les autres sains hommes faisoient en cellui temps. Berinus t. 2, 1350, p. 105

La locution constitue une unité assez soudée pour pouvoir se combiner avec si dans l'expression de la comparaison hypothétique :

9) Chascuns y puet aler ainsi que s'il n'avoit mal ne douleur. Jean d'Arras, Melusine, 1392, p. 114

1.1.2 Plus qu'une similitude entre le procès (action ou état) ou le sujet des deux verbes, de nombreux exemples démontrent une conformité du procès exprimé par la subordonnée avec un procès qui est de l'ordre de l'énonciation ou de l'attitude du locuteur à l'égard du dit :

10) Dame, quant serez vous m'amie / ainsi que le m'avez promis, / Si que je soie voz amis / De fait et d'oeuvre? Miracle de l'empereris de Romme, 1369, p. 263

11) laquelle, et par serement, sans aucune force ou contrainte, continua et persevera ès confessions cy-dessus escriptes, à li leues mot après l'autre, et icelles afferma par li avoir esté faites et cogneues ainsi que escriptes sont en ce present procès, et ne y sauroit que metre ne que oster. Registre Criminel du Chatelet t.1, 1389, p. 359

12) et oultre, se il a enffans de ma fille et ma fille meurt, ainsi que les choses pevent avenir, Froissart. Chroniques III, 1390, p.147

Les propositions ainsi que $q$ du type (2) peuvent être supprimées sans nuire à la grammaticalité de la phrase. Même si celles du type (1) ne peuvent l'être, il est clair qu'elles constituent plutôt des compléments circonstanciels, et non pas des compléments essentiels.

1.1.3 Mais contrairement aux emplois traités en (1.1.1) et (1.1.2), ainsi que q peut introduire un complément essentiel. Dans certains cas, il introduit le COD :

13) Bien avez oÿ comment Berinus fu esleüz a roy, et ainsi que Gieffroy s'en ala a Romme, et comment il fist forjurer a Logre le royaume de Blandie. Berinus t.1, 1350, p. 190

14) Et quant ce vint le landemain, cellui qui d'Orchaz estoit eschapé vif, si vint a l'empereur et lui compta mot a mot comment et en quelle maniere le corps estoit perdus et ainsi que cellui qui le print les deçut. Berinus t. 2, 1350, p. 17 
Coordonnant $q$ à d'autres propositions introduites par un interrogatif exprimant la manière (comment, en quelle manière), ainsi que a un fonctionnement de relatif comparable à celui de comme.

Par ailleurs, avec certains verbes, ainsi que $q$ introduit un complément qui, pour exprimer «la manière », n'en est pas moins un complément obligatoire; on traite quelqu'un (obligatoirement) de telle manière (sous peine que le verbe prenne un autre sens) $)^{7}$ :

15) Ce mesme jour, la Court a enjoint audit de Besze qu'il traicte sa femme amiablement et ainsi que mary doit traictier sa femme, et non autrement. Clement de Fauquembergue, Journal t. 1, 1421, p. 308

Dans cette occurrence, ainsi que q, coordonné à l'adverbe amiablement et opposé à non autrement indique «la manière » dont l'agent doit agir sur le patient dans la proposition régissante.

L'étiquette «comparaison » recouvre donc des fonctionnements syntaxiques et sémantiques différents, et l'on constate l'hétérogénéité de ainsi que en tant que MIS en MF.

La valeur temporelle, largement représentée, constitue un emploi supplémentaire de cette locution dans cet état de langue.

\subsection{La valeur temporelle}

Cette valeur, apparue au XIII ${ }^{\mathrm{e}}$ siècle $^{8}$, et bien attestée en MF est suffisamment stable, et indépendante des interprétations ou inférences contextuelles, pour que les dictionnaires du $17^{\mathrm{e}}$ siècle $^{9}$ l'enregistrent encore à côté de la valeur comparative ${ }^{10}$. Elle recouvre des relations de simultanéité (1.2.1) et d'antériorité (1.2.2), et peut même se teinter d'une nuance causale (1.2.3).

1.2.1. Toujours antéposée, $q$ exprime une action ponctuelle ou durative, présentée comme un arrière-fond, sur lequel on situe une action simultanée mais qui s'en

\footnotetext{
${ }^{7}$ La distinction complément essentiel vs complément circonstanciel se réfère surtout aux compléments directs et indirects; il ne serait pas inutile de creuser la notion de complément circonstanciel obligatoire, dans le domaine de la manière et du moyen. Pour un état de l'art, cf. Flaux \& Moline (2009).

8 «Et ainsy qu'ilz parloient ensamble en ce jardin, Becillas regarda au ciel» Roman des Sept Sages I, 2 (Imbs 1956 : 277)

${ }^{9}$ Cf par exemple Nicod, 1606 : Ainsi que, Tantost signifie circonscription de temps, comme en Amad. livr. 2. Et ainsi que l'on levoit les nappes, entra un gentil-homme tres-ancien, c'est à dire, au temps, ou tandis que l'on levoit les nappes, et tantost semblance de façon, au mesme livr. d'Amad. qui donnoient si grand clairté que l'on voyoit dedans la chambre ainsi qu'en plein jour, c'est à dire comme en plein jour.

10 Le sens temporel, s’il est jugé « vieux » par l'Académie, dès la 1ère édition de son Dictionnaire (1694) est maintenu dans l'édition de 1718 et n'en est retranché que dans celle de 1740 .
} 
détache, ce qui est marqué par l'emploi des temps (imparfait dans $q$ vs PS ou Pst dans $p)$ :

16) ainsi que Berinus et sa compaignie estoient en mer, il advint une vespree que une espesse nue se leva. Berinus t. 1, 1350, p. 208

17) Le lendemain, par matin, ainsi que le duc yssoit de sa messe et qu'il faisoit tromper pour deslogier et que l'avant garde s'estoit mise au chemin et le sommage, estes vous venus quatre chevaliers de la cité, armez et montez comme saint George Jean d'Arras. Melusine, 1392, p. 177

Dans d'autres cas, si le verbe de $q$, comme celui de $p$ sont à l'imparfait et expriment une action-durée, le premier relève du connu, sur lequel se détache le contenu informatif; ainsi que $q$ se réduit alors à une cheville syntaxique, selon un patron fréquent dans la prose médiévale ${ }^{11}$ :

18) ainsi que Berinus estoit en telle angoisse et en tel meschief, Aigres son filz l'attendoit au dehors de la tour. Berinus t. 1, 1350, p. 394

Dans tous les cas (16-17-18), on pourrait traduire la locution par « alors que ».

1.2.2. Par ailleurs, de la simultanéité dérive, dans certains contextes, une valeur d'antériorité immédiate, selon un mécanisme décrit, pour l'AF, par P. Imbs, et qui pourrait être étendu au-delà : «la postériorité n'étant, en ancien français, qu'une variante de la simultanéité - puisqu'elle se borne à noter la coïncidence du moment initial de l'action principale avec le moment où l'action subordonnée, à sa pointe extrême, arrive à son achèvement (et se situe, de par la partie achevée de sa durée, dans le passé révolu). Aussi la postériorité pouvait-elle s'exprimer par les morphèmes généraux de la simultanéité, qui ne sont en fait que des morphèmes de la coïncidence, la simultanéité et la coïncidence étant exprimées par l'accord ou le désaccord du temps et de l'aspect du verbe subordonné et du verbe principal» (Imbs 1956: 270). Le recouvrement du point d'arrivée de $q$ et du moment de départ de $p$ peuvent être marqués par l'opposition entre Passé antérieur/Plus-que-parfait et Passé simple; ainsi que se traduira alors par «quand, lorsque, au moment où » :

19) Et ainsi que li mariniers ot ceste raison comptee, Aigres estoit apoiez au bort de la nef, sy regarda devant lui et vit les ondes telles que cilz les avoit devisees. Berinus t. 1, 1350, p. 209-210

\footnotetext{
${ }^{11}$ Cf. Imbs (1956: 283) sur la proposition subordonnée antéposée qui résume une situation déjà exprimée : «Tous ces passages donnent l'impression d’un pur procédé de rhétorique, écho lointain de l'enseignement de Gorgias : la subordonnée en tête de phrase ou l'art des transitions! Transition par simple reprise d'une idée déjà émise, et qui par conséquent n'apprend rien; son rôle est purement formel ».
} 
Dans des contextes où $q$ comporte une périphrase inchoative ou la forme es vous (" voici»), la succession peut se teinter d'une idée d'immédiateté que l'on est tenté de traduire par « dès que, aussitôt que » :

20) Mais ainsi que les regarday, Il me conmencérent a rire. Miracle du Roy Thierry, 1374, p. 313

21) Et ainsi que ilz orent lavé et que ilz se deurent asseoir au disner, atant esvous venus deux chevaliers de la duchié de Lucembourc qui apportoient a Anthoine lettres de par sa moillier Crestienne. Jean d'Arras, Melusine, 1392, p. $193(\ldots)$

Ce qui est de l'ordre de l'implicature contextuelle a été donné d'ailleurs, à propos de si ...com, ainsi com, comme une valeur distinguant ces locutions du simple com(e) et l'assimilant à tantost com, sitost com (Imbs 1956 : 132).

1.2.3. Dans tel autre contexte, plus que de fournir un repérage temporel, ainsi que $q$ semble indiquer un événement qui est la cause de $p$ :

22) et quant ilz orent soupé, dirent li uns aus autres qu'ilz s'en alassent coucher au lieu que l'en dit le port au Fain de Paris, estant sur Saine, auquel lieu, ainsi que parolles se meurent entre lui et ledit Castille d'une part, et un des compaignons qui avoit soupé avecques lui, comme dit est, d'autre part, ycellui Castille fery d'un cousteau ycellui homme, et assez tost après ce, il qui parle de rechef fery sur ycellui homme, et tant qu'il le tuerent et lesserent pour mort en la place au Fain. Registre Criminel $d u$ Chatelet t.1, 1389, p. 11

Après une temporelle indiquant le cadre du récit («quant il eurent soupé »), la proposition au passé simple qui suit (ainsi que des parolles se meurent) semble bien exprimer le motif ( des paroles de querelles qui s'élèvent») de celle qui suit («le dénommé Castille frappa l'homme dont il est question»).

A la suite de ce qu'écrit Imbs (1956: 171) à propos de come, on pourrait suggérer que la distinction temporelle $v$ s causale n'est pas pertinente, la conjonction tendant à exprimer «les circonstances générales qui sont comme la manière de survenir de l'événement principal $»^{12}$. C'est sans doute ce caractère thématique qui explique la position systématiquement antéposée de ainsi que q à valeur temporelle.

\subsection{La valeur consécutive}

Cette valeur apparaît principalement dans des textes marqués de l'influence latine, que ce soit l'œuvre d'auteurs savants à proprement parler, comme les traductions d'Oresme, ou encore celle d'auteurs comme Christine de Pisan ou le chirurgien Martin de Saint Gille qui, sans être lettrés, n'en sont pas moins formés au

${ }^{12}$ Cf. également sur ce point Moline 2006. 
latin. On distinguera des cas problématiques où ainsi peut s'analyser comme un élément de la rection verbale (1.3.1) et des cas où le statut de locution de ainsi que est indiscutable (1.3.2).

1.3.1 Dans certaines occurrences, on peut hésiter sur l'analyse du syntagme ainsi que : faut-il vraiment y voir une locution conjonctive, [ainsi que] ouvrant une proposition $q$, ou doit-on analyser ainsi comme un constituant de la proposition $p$, exprimant la manière, précisé par une complétive $[q u e p]$ ? C’est en particulier le cas avec une proposition $q$ comprenant le verbe être qui appelle un complément :

23) Regularité et uniformité sont en mouvement et leur contraires aussi quant a son ysneleté, mais c'est differanment, car regularité et irregularité sont ou resgart du temps ainsi que se le mouvement est equalment isnel en chascune partie de son temps, il est regulier et se non, il est irregulier. Nicole Oresme, Le Livre du ciel et du monde, 1370, p. 410

24) Mais uniformité et difformité sont ou resgart du subject ainsi que se chascune partie du subject] meu est meue aussi isnelement une comme l'autre, le mouvement est uniforme et se non, il est difforme comment que il soit du temps. Nicole Oresme, le Livre du ciel et du monde, 1370, p. 410

25) Mais il n'en y ot nullui qui ne le ressoingnast, et longue piece fut ainsi que nul ne s' i osa enhardir de luitier. Berinus t. 2, 1350 p. 25

La double analyse est encore possible dans des phrases à $q$ négative :

26) Et Courroux qui estoit fier et orgueilleux, et qui avoit grant envie de ce qu'il avoit Aigres oÿ louer, quant il vist que Aigres mist debat au luitier, si en fu plus entalenté que devant et lui dist: «Comment, vassal, me fauldrez vous donc ainsi que pas ne luiterez a moy? » Berinus t. 1, 1350 p. 369

On peut d'autant mieux rattacher ainsi à $p$ que se rencontrent, à haute fréquence en MF, les structures il est ainsi, il advient ainsi, il dit ainsi où ainsi fonctionne comme une sorte de terme cataphorique développé par la complétive qui le suit ${ }^{13}$.

1.3.2 Toutefois cette analyse semble exclue dans des phrases où la valence verbale est déjà atteinte (27) ou dans celles qui comportent un terme marquant la manière (28) :

27) Et telz habis ne sont pas voluntaires, ainsi que l'en les puisse deposer quant l'en veult, mais eulz sont voluntaires pour ce que les operacions de

\footnotetext{
13 Il serait intéressant de comparer cet emploi de ainsi à celui de comme ça en français oral (p. ex. « Il a dit comme ça que... »; «puisque c'est comme ça que...» etc).
} 
quoy il furent causés estoient en nostre posté et volenté. Nicole Oresme, Le Livre de ethiques d'Aristote (Commentaire), 1370, p. 203

28) Une autre opinion fu que le ..ZZZVIIIe.. ciel la ou sont les estoilles fichies, est le souverain meu de simple mouvement journal et les autres cielx des planetes l'ensuient sans violence, ainsi que le plus prochain de lui l'ensuit plus et est plus tardif en son mouvement propre et le plus loing l'ensuit moins, comme devant dit est. Nicole Oresme, le Livre du ciel et du monde, 1370, p. 488

Dans ce cas, la traduction par «si bien que, de sorte que, de façon que » semble s'imposer et ainsi sort de la rection verbale, sans que l'on puisse dire si la locution est le résultat d'une réanalyse des structures précédentes ou un emploi de la locution comparative et temporelle dans le domaine consécutif, sur le modèle de si que, qui possède ces mêmes valeurs, selon une extension d'emploi favorisée par le moule latin sous-jacent ita ut, sic ut. Cet emploi consécutif fait très majoritairement apparaitre un verbe au subjonctif en $p$, sans que ce soit une règle générale (31):

29) Dire que c'est possible que une chose soit faite de nouvel et que elle soit incorruptible ou perpetuelle et que une chose qui a duré sanz conmencement soit apres corrompue, ainsi que une chose ait une foys generacion sans avoir corrupcion ou que une autre ait une foiz corrupcion sans avoir [ eu ] generacion, ce dire est interimer aucunes des choses devant suppousees. Nicole Oresme, le Livre du ciel et du monde, 1370 , p. 230

30) Es agues continues, se la levre, l'euil, le sourcil, ou le nez soient teurs, ainsi que le pacient ne voye point, la vertu du corps affoiblie et debilitee, se toutes ces choses sont faictes, le pacient est prez de la mort. Martin de Saint-Gille, Les Amphorismes Ypocras, 1362, p. 76

31) Mais, pour faire li contes cours, Vous di que moult bien s'i porterent Li Grec, ainsi que maint porterent Des Troyens a terre, sans faille, En frappant d'estoc et de taille. Christine de Pizan, Le Livre de la Mutacion De Fortune t. 3, 1400, p. 78

On aura constaté dans ce point (1) la diversité sémantique et syntaxique des propositions introduites par ainsi que.

\section{Ainsi que comme introducteur de construction elliptique}

Les constructions elliptiques introduites par ainsi que sont à mettre en rapport avec la comparaison, et pourraient être traitées sous cette rubrique. On a toutefois pris le parti de les décrire indépendamment pour deux raisons : d'une part, il a été noté 
(Desmets $2008^{14}$ prolongeant Ginzburg \& Sag 2001 et Culicover \& Jackendoff 2005) que les constructions comparatives elliptiques présentent des traits interdisant de les ramener à une transformation de la proposition comparative, ce qui nécessite de traiter ces « séquences fragmentaires » comme un type de séquence spécifique ; d'autre part, le mécanisme de comparaison, supposant la mise en rapport de deux entités distinctes, peut aboutir, et c'est le cas en MF, à des valeurs d'approximation ou d'exemplification qui s'écartent de la comparaison proprement dite. Par ailleurs, la valeur coordonnante (Pierre ainsi que Jean appuient la proposition; Il condamne l'agresseur ainsi que la victime), parente de la comparative elliptique, n'en présente pas moins des caractéristiques distinctes (Desmets 2008). La valeur coordonnante, actuellement sans doute la plus vivante en français, n'apparaît qu'exceptionnellement dans le corpus de MF, et une étude ultérieure est nécessaire pour décrire le glissement des séquences fragmentaires comparatives à des séquences de coordination. Le classement des structures elliptiques en ainsi que présentée ici, en fonction du nombre d'éléments résiduels de la séquence, en constituera un utile préalable.

On observera donc successivement les séquences fragmentaires comportant deux « éléments résiduels » (Sujet-Objet direct/indirect; Sujet-Attribut ; Sujet-Complément prépositionnel instrumental) (2.1), puis celles qui en comporte un seul (Sujet, Complément Prépositionnel et Adverbe) $(2.2)^{15}$. On s'interrogera enfin sur le statut de séquences en ainsi que faisant apparaitre des valeurs d'approximation et d'énumération, et plus exceptionnellement de coordination (2.3).

\subsection{Séquence fragmentaire à deux éléments résiduels ("phrase trouée »)}

La «phrase trouée » thématise la relation entre deux arguments de la comparaison tout en omettant le prédicat verbal. Les deux éléments résiduels sont donc le sujet et l'objet direct ou indirect, et aussi le sujet et l'instrumental, mais la phrase trouée peut présenter des modifications quant à la linéarisation (32-33) ou la construction du prédicat (34-35), qui empêchent d'en faire une simple réduction de ce que serait la proposition subordonnée :

32) Adonc les gentilz hommes de la nacion de Bretaigne comme tres resjois pristrent à venir de toutes pars le suivre et avironner tout ainsi que la geline les poscins. Christine de Pizan, Le Livre des Fais et bonnes meurs $d u$ sage roy Charles $V, 1404$, p. 188

33) Car se les gentilhommes feissent Aussi bien que nous leur debvoir, Que le roy des corps ilz servissent ainsi que nous de nostre avoir, Les estrangiers pas ne pillassent. Alain Chartier, Le Debat de Herault, du vassault et du villain, 1415, p. 434

\footnotetext{
${ }^{14}$ Desmets (2008) prend pour objet les séquences fragmentaires en comme du FM qui posent des problèmes analogues, à partir de la valeur comparative.

15 On parlera dans le premier cas de "phrase trouée» (gapping), dans le second de «construction différée » (stripping), selon Desmets (2008) prolongeant Ginzburg \& Sag (2001).
} 
34) Ceulx le scevent qui l'ont essayé, combien que parler n'en puis - dont il me poise -, ne mais ainsi que l'aveugle des couleurs. Christine de Pizan, Le Livre des Trois Vertus, 1405, p. 23

35) Puis qu'a amer pris, ne cessay, N'oncques puis Penser ne lessay, Qui son couvent Ne tient, maiz le tourne souvent, ainsi que le cochet au vent. Alain Chartier, Le Livre des quatre Dames, 1416, p. 249

Dans (32) la linéarisation fait apparaittre en q l'objet (la geline « la poule ») avant le sujet (les poscins « les poussins »), à la différence de l'ordre de la phrase matrice tandis que dans (33) le complément de moyen (des corps) antéposé au sujet (ils) dans la matrice se trouve postposé au sujet dans la séquence fragmentaire (nous de nostre avoir), créant un chiasme. Dans l'exemple (34) il semble que se télescopent la comparaison d'égalité ( je ne peux pas en parler de même que l'aveugle ne peut pas parler des couleurs ») marquée par ainsi que et la disparité (je ne peux pas en parler, pas plus que l'aveugle ne peut parler des couleurs) marquée par la négation et l'adverbe mais ("davantage »). Dans (35) la séquence fragmentaire joue des deux constructions propres à un verbe symétrique comme tourner: l'intention tourne (= fait tourner) son engagement comme la girouette (tourne) sous l'effet du vent.

Ces phrases trouées, qui sont somme toute en nombre limité dans le corpus, seraient à dériver uniquement des comparatives marquant la similitude étudiées précédemment (1.1.1).

\subsection{Séquence fragmentaire à un élément résiduel (" construction différée »)}

L'élément résiduel peut être le Sujet grammatical (2.1) ou, plus généralement, le thème (2.2), ou bien un Complément Prépositionnel ou un Adverbe (2.3) à valeur circonstancielle (temps et lieu).

2.2.1 Quand l'élément résiduel est le Sujet, la séquence fragmentaire correspond majoritairement aux propositions constituant un complément nécessaire de $p$ : à l'exception de (38) où la séquence, intercalée entre verbe et complément, pourrait être supprimée sans nuire à la bonne formation de la phrase, ainsi que p, en tant qu'expression de la manière ne peut être omis. La séquence fragmentaire vient en général en seconde position (36-37), mais elle peut aussi s'intercaler entre des constituants de la phrase matrice (38) :

36) Maiz vivez ainsi que une beste, Si en vendrez a beaux chevaulx. Estoire de Griseldis, 1395, p. 45

37) mais, par la machinacion d'aucuns autres officiers, la ville et chastel, sans sa coulpe, fu baillée aux ennemis, et combien qu'il se soit tousjours gouverné ainsi que ung noble homme loyaument, neantmoins on a voulu chargier son honneur. Clement de Fauquembergue, Journal t. 2, 1421, p. 348 
38) S'ilz se fussent tousjours tenu, ainsi que vous, blanc, moyte et chault, L'onneur ne leur fust pas venu, Car on n' a jamaiz bien sans peyne. Alain Chartier, Le Debat de Herault, du vassault et du villain, 1415, p. 423

On notera que le verbe qui serait restitué dans une subordonnée complète ne pourrait apparaître à la même forme que dans la phrase matrice en cas de verbe à un mode non fini :

39) comment les sains prelas du temps passé n'aquistrent pas paradis par faire ainsi que les prelas de maintenant. Deschamps, le Miroir de mariage, $1385: 162$

2.2.2. Dans le cas d'une prédication existentielle, avec le verbe copule être (40) ou la périphrase il y a (41), la séquence fragmentaire qui présente le thème, est suppressible :

40) Des chienz, comme j'ay dit sa devant, sont les uns plus saiges que les autres einsi que des hommes, quar il y a tieulx chienz, pour quant qu'ilz eussent bon maistre, jamais ne seroyent saiges et tieulx qui seront saiges en une sayson. Gaston Phebus, Livre de Chasse, 1387, p. 209

41) Et ce leur vient de trois choses : l'une, pour ce qu'ilz ont bon nés, l'autre, de la grant volenté qu'ilz ont, la tierce, il y a des chienz parlerres et janglerres et estourdiz einsi que des genz. Gaston Phebus, Livre de Chasse, 1387, p. 210

2.2.3. De même, quand l'élément résiduel est un adverbe ou un complément prépositionnel à valeur temporelle ou faisant référence à un passage antérieur, la séquence pourrait être supprimée sans nuire à la bonne formation de la phrase :

42) Et finablement la Court, à l'instance des assistens, encharga aux dessusdis commissaires que en perseverant ilz assemblassent, ainsi que par avant, diligemment et continuelment pour entretenir le fait de ladicte commission. Clement de Fauquembergue, Journal t. 1, 1421, p. 233

43) Et n'est point acoustumé d'appeller de leurs jugemens, et n'ont point acoustumé de prendre ressort en la Chambre de Gand, ne ailleurs, et n'y ressortissent, mais ont acoustumé de terminer et mettre fin en tous les procès de leursdis bourgois et subgiez, en usant de leurs privileges, usages, coustumes et prerogatives qui leur sont neccessaires, et leur est besoing de les garder pour l'entretenement et conservacion de leur dicte ville et de leurs bourgois et subgiez, qui sont rondes gens et vivent rondement, et n'ont mie acoustumé de plaidier longuement, ne delaier leurs procès, ainsi que en aucuns autres lieux de ce royaume, où il y a autres usages et coustumes selon l'exigence et la neccessité des païs. Clement de Fauquembergue, Journal t. 2, 1421, pp. 297-298 
Ces séquences fragmentaires rejoignent les propositions complètes du type 1.1.2 : elles sont suppressibles, et introduisent une information connue.

\subsection{Séquence fragmentaire à un élément résiduel et syntagme intraphrastique}

Réduite à un élément, la séquence fragmentaire tend à se rapprocher d'un syntagme nominal intraphrastique, complément nécessaire du verbe (2.3.1), en passant éventuellement de la comparaison à l'approximation, au point que la «locution conjonctive» peut fonctionner comme un marqueur d'approximation; la perte du caractère propositionnel est atteinte dans des emplois d'approximation, où disparaît le terme de comparaison $p$, ainsi que ayant alors un fonctionnement de préposition (2.3.2) introduisant un ajout circonstanciel, tandis que dans la valeur d'exemplification (2.3.3), la séquence fonctionne comme apposition à un syntagme de $p$ et non par rapport à la prédication. Cette restriction de portée apparaît également dans l'occurrence (unique) à valeur d'ajout coordonnant.

2.3.1. Dans certains cas, contrairement aux constructions étudiées en 2.2.3, la séquence fragmentaire, quoique constituée d'un Complément Prépositionnel, entre dans la construction du verbe et ne peut être supprimée ; c'est le cas des constructions où le verbe fait attendre l'expression de la manière (44) :

44) Si a la Court appoincté que le procureur du Roy mette devers la Court arrestz, ordonnances, lettres, citacions et ce que vouldra, et ladicte Court les verra, et en ordenera ou appoinctera ainsi que de raison. Clement de Fauquembergue, Journal t.2, 1421, p. 231

Parmi les emplois où la séquence ainsi que $X$ n'est pas suppressible, on doit distinguer les occurrences où $p$ comporte le verbe être comme (45):

45) Pour touz amans mettre en joie a durer, Le tresdoulx corps de la vierge Marie Reserva Dieux ou plus hault throsne cler, Pour estre ainsi que une porte jolie En qui l'engien divin par sa pitié Descendre fist. Le Miracle de l'enfant ressuscité, 1353, p. 81

Cet emploi se distingue, en effet, aussi bien au plan sémantique que syntaxique : d'une part, la comparaison porte sur le prédicat de $p$, au lieu de constituer, comme dans les autres emplois, elliptiques ou non, un commentaire sur l'énonciation de $p$ ou la mise en équivalence de deux prédications ${ }^{16}$; d'autre part, la locution ainsi que est

\footnotetext{
${ }^{16}$ On reprend en particulier ces distinctions de Pierrard et alii (2006:136), qui l'illustrent des exemples suivants :

- Portée énonciative : Ainsi que/ comme/*de même que/*tel que/*aussi bien que son nom l'indique, monsieur Pain est boulanger; Pierre est / comme / ainsi que/*de même que/*tel que/*aussi bien que disait mon voisin, une fripouille.
} 
suppressible, sans nuire à la bonne formation syntaxique de la phrase, mais en transformant le figure de comparaison en métaphore.

Du fait que la comparaison repose sur la mise en rapport de deux prédicats, elle peut exprimer une coïncidence quasi parfaite comme en (45), mais aussi une équivalence approximative si le recouvrement est partiel comme en (46-47) :

46) Saint Augustin dit que cellui Naxagoras fu condempnez à Athenes pour ce que il disoit que le soleil n'estoit autre chose que ainsi que une pierre ardent, et ceulz d'Athenes aouroient le soleil comme Dieu. Christine de Pizan, Le Livre des Fais et bonnes meurs du sage roy Charles V, 1404, p. 64

47) Sus ce pourpos ilz furent ainsi que d'accort, et sus le point que de faire leur fait droit là, quant aulcuns des cappitaines se raviserent et mistrent en termes en disant: "Clermont est une puissant ville et fort peuplée, et les gens bien pourveus d' armures. Froissart, Chroniques III, 1390, p. 211

Le soleil ressemble certes à une pierre en fusion, mais il ne se résume pas à cela, il est «une sorte de pierre en fusion»; les adversaires sont quasiment d'accord pour régler un différend, mais certains se ravisent et ils ne le font pas, ils n'étaient que "presque d'accord». Le MIS ainsi que rejoint donc là la catégorie des marqueurs d'approximation ${ }^{17}$, et l'on peut s'interroger sur le statut de l'élément qui le suit : peuton vraiment parler d' "élément résiduel» (d'une proposition q), ou doit-on le considérer comme un syntagme nominal constituant de $p$ ?

2.3.2. C'est sans doute de ce type de construction que sont issus les emplois où la séquence fragmentaire introduit un élément qui n'a pas de comparant strict dans la phrase matrice :

48) Mais Aigres, qui avoit grant soubtiveté en lui, leur fist faillir a leur esme, car lors qu'il fut revenu de Moreau l'ermite, ainsi que sur le point de l'adjourner, il desarma sa mere et sa suer. Berinus t. 1, 1350, p. 415

49) Et, s'il avenoit qu'ilz menjassent et s'en alassent hors dou buisson, et cela ilz faisoient par deux ou par trois nuiz, sanz que null n'i demourast, il doit au vespre, devant qu'il soit nuit, pendre la charoigne par les arbres si haut que lou n'i puisse avenir et laissier des os, s'il en y a, en terre, affin qu'ilz les rungent, et venir au buisson einsi que une heure devant le jour. Gaston Phebus, Livre de Chasse, 1387, p. 239

- Portée prédicationnelle : (Préd 2 porte sur l'ensemble Préd 1) : Comme/ainsi que/ de même que/ *tel que/ aussi bien que les amateurs de vin parlent en Millésimes, les. Anglais mesurent la valeur de leur jeunesse universitaire aux performances des équipages.

- Portée prédicative : Elle est belle comme/*ainsi que/*tel que/*de même que une fée.

${ }^{17}$ Cf. Lakoff G., (1973) ; Bat-Zeev Shyldkrot, Adler \& Asnes (2010) ; Bat-Zeev Shyldkrot (à par.) ; Bertin (à par.). 
50) Quant les Anglois eurent fait leur emprise et courut devant la ville, et ilz sentirent que les François s'ordonnoient pour venir sus eulx, si rapasserent tout bellement le rieu que passé avoient, et se retrairent tout en brouse sus un grant sablonniere qui là estoit et eslongierent le rieu, ainsi que le trait de troix arcz arriere. Froissart, Chroniques III, 1390, p. 94-95

51) Et la doit fere une fousse et gieter la charoigne dedanz et leissier un pertuis en l'une part de la fousse, einsi que le grant de la teste d'un homme. Gaston Phebus, Livre de Chasse, 1387, p. 266

52) Et se doivent mettre dedanz le bois au couvert einsi que une toyse dedanz, loing l'un de l'autre le giet d'une petite pierre. Gaston Phebus, Livre de Chasse, 1387 , p. 288-289

Ainsi que signale le caractère approximatif de l'indication de temps ou d'espace qui suit. Si l'on veut percevoir une comparaison, il faut restituer le terme de comparaison à partir d'un élément du contexte gauche indiquant le temps (lors que; au vespre, devant qu'il soit nuit) ou l'espace (eslongier; pertuis), ce qui devient impossible quand la séquence en ainsi que est en position initiale de la phrase :

53) Ainsi que une lieue de Clermont, ilz rencontrerent Aymerigot Marcel et bien cent lances, lequel estoit cappitaine de la garnison d'Aloise delez Saint-Flour. Froissart, Chroniques III, 1390, p. 209

Cette dernière occurrence confirme le fait que la catégorisation comme préposition à valeur d'approximation se fait en fonction du sémantisme du SN de droite, qu'il exprime le temps (sur le point de l'adjourner, une beure devant le jour) ou l'espace (le trait [d'un arc], le grand [« la taille»] d'une teste, une toyse, une lieue) plus que du contexte gauche. Dans ce cas, le syntagme semble avoir perdu son statut de séquence fragmentaire (de proposition) pour constituer un syntagme complément prépositionnel au statut d'ajout. Le MIS à valeur de marqueur d'approximation, s'il précède un SN sans préposition (49-52) prend alors lui-même valeur de préposition ("à environ »).

2.3.3. Ainsi que peut enfin marquer une relation d'identité entre un syntagme de $p$ et $q^{18}$; la séquence ainsi que $q$ est en l'occurrence suppressible. Dans (54), le SN qui constitue $q$ sert ainsi à exemplifier le pronom $c e$ :

54) Tu t'en iras droit en Egipte A un saint hermitte qui sert Jhesu Crist, et en un desert S'est mis pour le monde eschiver Et vit de ce qu'il peut trouver Par le bois, ainsi que racines Et fruiz de ronces et d' espines, Et poires et pommes sauvages; Miracle de un paroissian esconmenie, 1356, p. 34

Dans (55) et (56), la séquence entre en relation avec un complément déterminatif du GN sujet ou thème :

18 Cf. Pierrard \& alii (2006 : 137). 
55) De touz poillz de chienz courranz y a de bons et de mauvais einsi que de levriers et d'autres chienz, mes le plus commun poill qui soit bon de chienz courranz, si est noire catruillé. Gaston Phebus, Livre de Chasse, 1387 , p. 129

56) Aussi bonté de chien courrant einsi que de toutes autres natures de chienz vient de droit cuer et de bonne nature, de bon pere et de bonne mere. Gaston Phebus, Livre de Chasse, 1387, p. 129

On notera qu'en (56) la substitution de la coordination et au MIS est possible, c'est le seul cas rencontré.

La construction et la valeur de ainsi que q sont apparues très variées, aussi bien en construction elliptique que lorsque $q$ constitue une proposition complète. On doit confronter cette diversité aux emplois contemporains afin d'interroger l'évolution de la conjonction.

\section{Questions sur l'évolution des conjonctions}

Une forte proportion des constructions de ainsi que du MF ont disparu. Après l'avoir mis en lumière (3.1), on envisagera les causes de ce qui peut paraittre un blocage à la grammaticalisation d'un morphème qui, malgré son caractère polymorphémique, semblait apte à concurrencer le morphème héréditaire comme monomorphémique, dans la variété de ses emplois et de ses valeurs. On devra se demander si cette régression des emplois autres que comparatifs correspond à une "grammaticalisation avortée », ou si, au contraire elle dénote une grammaticalisation accrue (3.2), ce qui invite à réfléchir sur la diversité du mécanisme de la grammaticalisation dans le domaine de la conjonction (3.3).

\subsection{Bilan- Les emplois de ainsi que en MF et en français moderne}

La comparaison des emplois en MF et en FM fait apparaittre la disparition des valeurs temporelles (de la simultanéité à l'antériorité immédiate) et consécutives (1.2 et 1.3), et celle de la valeur d'approximation (2.3) qui tendait à faire glisser ainsi que de la catégorie de la conjonction vers celle de la préposition, c'est-à-dire une catégorie de morphèmes assurant une intégration à l'échelle du syntagme et non plus de la proposition (2.3). Parmi les séquences fragmentaires à valeur proprement comparative, un nombre important des séquences à un ou deux termes résiduels ont disparu, aussi bien l'ensemble des «phrases trouées » où ainsi que q thématise la relation de similitude que les «constructions différées» où la séquence thématise l'un des arguments de la similitude ou exprime le contexte situationnel (2.1 et 2.2). En revanche, la valeur d'ajout du MIS, faisant passer la conjonction de la subordination à la coordination, pour autant que ces catégories soient valides, s'est considérablement développée audelà du MF, l'étude détaillée de ce glissement restant à faire. 
L'écart semble donc important ${ }^{19}$. Toutefois on remarquera la stabilité des caractéristiques prédominantes de la construction du MIS, selon les trois points de vue de la hiérarchisation ${ }^{20}$, de l'autonomie prédicationnelle, liée à la perte du statut de proposition $^{21}$, du degré \pm fort de la rection verbale selon le type de verbe ${ }^{22}$. L'étude menée pour les MIS du Français Moderne par Pierrard \& al. (2006) dégage l'affinité de ainsi que avec les caractéristiques d'une intégration moyenne, plus faible que celle du MIS tel que, par exemple. Selon ces auteurs, du point de vue de l'opposition autonomie $v$ s hiérarchisation ${ }^{23}$, l'adjonction (Pierre appuie la proposition ainsi que Jean) l'emporte sur la corrélation (Ainsi que l'ébéniste agence ses pièces de bois, ainsi l'écrivain agence ses mots) ou la subordination (il a agi ainsi qu'il l'a toujours fait), et prédominent la portée énonciative (Ainsi que son nom l'indique, Monsieur Pain est boulanger) et la portée prédicationnelle (Ainsi que les amateurs de vin parlent en Millésimes, les Anglais mesurent la valeur de leur jeunesse universitaire aux performances des équipages), alors que les portées plus étroites, prédicative (*Elle est belle ainsi qu'une fée) ou syntagmatique $\left({ }^{*} U n\right.$ homme ainsi que toi ne fait pas des choses aussi vulgaires) sont exclues. En ce qui concerne la force \pm grande de la rection verbale, ainsi que « préférerait des prédicats à rection plus légère, tout en évitant les structures prédicationnelles réduites, sauf dans le cas de la syntagmatisation » (ibid.140); la rection par un verbe lexical plein est possible (Il est ressorti ainsi qu'il était entré ${ }^{24}$, mais les verbes à rection plus lâche, verbe de dire ou de perception (Ainsi que disent les braves gens, il bat la campagne), verbe modal (Ainsi que le voulait la coutume, il fit d'abord une révérence à la déesse Soleit), pro-verbe (il a agi ainsi qu'il l'a toujours fait) prévalent. De plus, ainsi que montre une prédilection pour les prédicats décolorés, constitués d'une reprise du prédicat principal (Ainsi que trop d'impôt tue l'impôt, trop d'Etat tue l'Etat) ou d'un pro-verbe (Il est ressorti ainsi qu'il faisait d'habitude), et il permet la syntagmatisation du thème (Pierre ainsi que Jean appuient la proposition; Il condamne l'agresseur ainsi que la victime) ; ces deux points le distinguent de tel que, mais, en FM du moins, il ne tolère pas que le prédicat se réduise à une relation thématisée (? Il connaît le latin ainsi que le prêtre son bréviaire) ni à un argument thématisé de la relation de similitude (*Il a bondi ainsi qu'un lion; * Une femme ainsi que sa mère ne pleure pas) ni à un

\footnotetext{
${ }^{19}$ Voir tableau en annexe 1, récapitulant la comparaison des deux états de langue selon les critères mentionnés ici.

${ }^{20}$ La hiérarchisation est fonction du degré d'autonomie de $p$ et $q$ (selon un ordre décroissant: cohésion $>$ adjonction $>$ corrélation $>$ subordination) et du niveau de portée syntaxique (du plus large au plus étroit: portée énonciative $>$ portée prédicationnelle $>$ portée prédicative $>$ portée syntagmatique); Cf. Pierrard et alii (2006, 134-138).

${ }^{21} \mathrm{La}$ perte du statut de proposition est marquée, selon un ordre croissant, par le fait que $q$ est constitué par : un "prédicat décoloré » (répétition du prédicat ou proverbe) ou, par rapport à p, une relation thématisée < un argument thématisé < une expression du contexte prédicationnel < une syntagmatisation du thème ; Cf. ibid., 138-139).

${ }^{22} \mathrm{La}$ force de la rection verbale est décroissante selon l'ordre : verbe lexical $>$ verbe de dire ou de perception $>$ verbe modal > pro-verbe (ibid., 139-140).

23 "Ainsi (que) et de même (que) ont un spectre d'emploi large mais plutôt du côté du pôle «autonomie » et plus ancrés dans le cohésif et l'énonciatif, ce qui semble lié à leur morphologie analytique (forme complexe) » ibid., 137).

${ }^{24}$ Les auteurs indiquent que cet emploi est plus contraint, ce qui nécessiterait d'être précisé.
} 
élément thématisé du cadre (*Il chante ainsi que dans sa jeunesse; *Il se bat ainsi que quand il était jeune; *Il se bat ainsi que s'il était jeune). On constate bien des points communs avec les caractéristiques dégagées par l'observation de notre corpus quant aux types de verbe représentés dans $q$ (pro-verbe, verbe de dire et de modalité plutôt que verbe lexical plein). On peut alors se demander si en éliminant certaines structures et certaines valeurs, dont la valeur consécutive, l'évolution diachronique n'a pas en fait développé les caractéristiques fondamentales du lien instauré par le MIS ainsi que, soit une relation sémantique peu saillante correspondant à une faible intégration syntaxique.

\subsection{L'évolution de ainsi que : grammaticalisation avortée ou accrue?}

La grammaticalisation des conjonctions et locutions conjonctives semble suivre un parcours évolutif général qui aboutit à l'expression des relations logiques (causecondition-concession) à partir de l'expression de relations temporelles et de relations modales (Kortmann 199725, Bat-Zeev Shyldkrot 1988). Comme (< latin quomodo, sur modus « la manière »), forme simple héritée du latin, connaît tout le spectre d'emplois, du modal (comparaison, approximation, manière) au temporel et au logique (causal). Or l'histoire de ainsi que montre, qu'après avoir connu un élargissement similaire de ses emplois, son sémantisme s'est restreint au domaine de la modalité. Aussi Pierrard \& al. (2006 : 143) avancent-ils que "Pour ainsi que il n'y a pas de grammaticalisation vers des valeurs de conséquence », ce qu'ils illustrent par «La situation a évolué *ainsi qu'/de telle façon que/de manière à ce qu'on n'aura plus besoin d'aide ». Une telle grammaticalisation pourtant a bien eu lieu, on a l'a vu (infra 1.3).

Faut-il voir là un cas de "grammaticalisation avortée »? Plusieurs facteurs pourraient tout à la fois amener à l'affirmer et à l'expliquer. En premier lieu, le fait que les conjonctions exprimant le champ de la modalité connaissent davantage des glissements de sens à l'intérieur de leur domaine (de la manière à l'instrumental, ou de la manière à la comparaison, par exemple), et moins fréquemment un glissement à l'extérieur de leur domaine, vers l'expression du temps puis des relations logiques (Kortmann 1997 : 196). Plus spécialement, on l'a vu, la valeur consécutive est un fait de langue savante; même si elle a pu se développer spontanément par réanalyse d'énoncés où ainsi, dans la rection du verbe principal, se trouvait, suivi d'une complétive, cette valeur a pu être sentie comme un emprunt syntaxique au latin sic...ut et, en tant que tel, un élément artificiel et allogène. En tout état de cause, même produite par une réanalyse spontanée, la valeur consécutive ne semble pas s'être diffusée à la langue générale, ce qui manifesterait le peu d'aptitude à la grammaticalisation de cette locution en conjonction à valeur logique.

Par ailleurs, certains emplois exprimant une valeur d'approximation (infra 2.3) pouvaient amener à une recatégorisation en marqueur d'approximation ("presque ») voire en préposition (« environ à »), faisant passer le morphème du grammatical (la locution conjonctive) à du plus grammatical (marqueur et préposition), en tant que la portée du marqueur et de la préposition est plus restreinte, ce qui est l'un des critères du degré de grammaticalisation (Lehmann 1985). Cette polyfonctionnalité, qui allait

${ }^{25}$ Cf. en particulier ch. 7, tableau récapitulatif, p. 210. 
contre une tendance forte de l'évolution du français (Marchello-Nizia 2006), ne s'est pas développée, différenciant là encore au fil du temps la conjonction de forme analytique et la forme héréditaire comme dont certains emplois semblent bien relever de ces catégories ${ }^{26}$.

La grammaticalisation de la locution ainsi que, de ce double point de vue, semble donc régresser. On doit pourtant se demander si en éliminant les sens non prototypiques (temps, conséquence, approximation) l'évolution n'a pas développé le sens virtuellement premier de la locution, à savoir une mise en relation thématique sans caractère sémantiquement saillant. Si la javellisation du sens qui accompagne la grammaticalisation permet de dégager des traits sémantiques d'une grande abstraction, en débarrassant le terme de tous les sens accessoires, d'ordre lexical, qui lui donnaient sa spécialisation sémantique (Sweetser 1988), on peut dire que la locution comparative, qui prend majoritairement une valeur de copule additive, connaît un accroissement de grammaticalisation : la mise en rapport impliquée par l'acte de comparer se ramène à un schéma, plus général, de sérialisation, de liste.

\subsection{Conclusion-Réflexions sur la grammaticalisation des locutions conjonctives}

La grammaticalisation d'une conjonction est donc à considérer à différents niveaux. On peut en effet se demander pourquoi des conjonctions issues du même domaine sémantique source évoluent de manière différente. La «loi de répartition» que constitue la tendance à l'iconicité y joue certainement un rôle. Mais le sémantisme du terme-source est également un facteur déterminant.

L'évolution divergente de ainsi que (qui se spécialise dans le domaine comparatif puis la coordination) et de si...que (de la comparaison à la conséquence) peut être mise en relation avec le fait que le morphème si perd son statut d'adverbe thématisant, qui était de haute fréquence en ancien français et en moyen français, pour se spécialiser dans l'expression de l'intensité27, alors que ainsi fonctionne très majoritairement par rapport à un antécédent du discours. Pour rendre compte de l'évolution des locutions conjonctives, on constate la nécessité de prendre en considération tout à la fois les relations sémantiques très générales (mode, temps, lieu, relations logiques) qu'elles marquent et les mécanismes discursifs et énonciatifs qu'elles signalent.

L'étude d'un corpus historiquement limité d'une locution particulière amène ainsi à s'interroger sur le rapport de l'évolution diachronique des locutions conjonctives à un sens prototypique, en relation avec la persistance sémantique du constituant qui leur sert de base.

\footnotetext{
26 Cf. en particulier Halmøy 1998.

27 On rapprochera cette évolution de celle des locutions formées sur un élément à l'origine phorique (tel, tant) ayant développé au fil des siècles une valeur intensive.
} 


\section{RÉFÉRENCES BIBLIOGRAPHIQUES}

BAT-ZeEv ShyldKrot, H., \& KEMmer, S., 1988, «Le développement sémantique des conjonctions en français: Quelques concepts généraux », Revue Romane, 23.1, p. 9-20.

BAt-Zeev Shyldkrot, H., Adler, S., \& Asnes, M., (éd.), 2010, « Approximation et précision : un champ à explorer », L'Information grammaticale, 125, p. 3-5.

BAT-ZEEV SHYLDKROT, H., (à par.): «Approximation et précision : états des lieux ».

BERTIN, A. (à par.) : «Parcours sémantique du mot assez: réflexions sur un intraduisible du français médiéval ».

Culicover, P., \& JACKENDOFF, R., 2005, Simpler Syntax. Oxford University Press.

Desmets, M., 2008, «Ellipses dans les constructions comparatives en comme», Linx, 58, p. 47-74.

Delabre, M., 1984, «Les deux types de comparaison avec comme», Le Français Moderne, $52 \frac{1}{2}$, p. $22-47$.

Flaux, N., \& Moline, E., 2009, «De la manière », Présentation de Langages, 175, p. 3-14.

GINZBURG, J. \& SAG, I.A., 2001, Interrogative investigations : the form, meaning and use of English interrogatives. Stanford, CSLI Publications.

HaLmøy, O., 1998, «Comme : adverbe, conjonction...et préposition ?», in M. Bilger, K. Van den Eynde \& F. Gadet (éds), Recueil d'études offert en hommage à Claire BlancheBenveniste, Orbis Supplementa 10, Leuven-Paris, Peeters, p. 221-228.

Haspelmath, M., \& BuCHOlZ, O., 1998, «Equative and similative constructions in the languages of Europe », in van der Auwera, J. (éd.), Adverbial constructions in the languages of Europe. Berlin, Mouton de Gruyter, p. 277-334.

IMBS, P., 1956, Les Propositions temporelles en Ancien Français. La détermination du moment, Strasbourg, Publication de la Faculté des Lettres de Strasbourg.

JONAS, P., 1971, Les systèmes comparatifs à deux termes en ancien français, Bruxelles, Editions de l'Université de Bruxelles.

Kortmann, B., 1997, Adverbial Subordination. A typology and History of Adverbial Subordinators Based on European Languages, Berlin-New York, Mouton de Gruyter.

LAKOFF, G., 1973, "Hedges: A study in meaning criteria and the logic of fuzzy concepts », Journal of Philosophical Logic 2 (1973), p. 458-508.

LeHManN, C., 1985, "Grammaticalization: Synchronic Variation and Diachronic Change », Lingua e stile, XX-3, p. 303-318.

LEHMANN, C., 1988, «Towards a typology of clause linkage », in Haiman, J. \& Thompson S. A. (éds.), Clause Combining in Grammar and Discourse, Typological Studies in Language 18, Amsterdam, John Benjamins. 
LÜRH, R., 1989, «Ist die Basiskategorie TEMPORAL als Ausgangspunkt für konjunktionale Bedeutungen ein Universale? », Historische Sprachforschung 102, p. 153-173.

MARCHELlo-Nizia, C., 1985, Dire le vrai : l'adverbe d'assertion si en français médiéval. Essai de linguistique historique, Genève, Droz.

MARCHELlo-NizIA, C., 2006, Grammaticalisation et changement linguistique, Bruxelles, de Boeck.

PIERRARD, M., HADERMANN P., van RAEMDONCK D., 2006, «Les marqueurs d'identité : subordonnants, coordonnants ou corrélateurs? », Faits de langue 18, p. 133-144.

MOLINE E., 2006, «Et comme minuit allait sonner, ...Pour en finir avec la partition temporelles us causales », Cabiers Chronos 15, p. 63-90.

SWEETSER E., 1988, "Grammaticalization and semantic bleaching », Berkeley Linguistics Society 14, p. 389-405.

\section{Bases de données}

BFM : Base de Français Médiéval, [en ligne]. Lyon: UMR ICAR/ENS-LSH, 2006. Site internet : http://bfm.ens-lsh.fr.

DMF : Dictionnaire du Moyen Français. ATILF / Nancy Université - CNRS. Site internet : http://www.atilf.fr/dmf.

\section{ANNEXE}

\begin{tabular}{|c|c|c|c|}
\hline $\begin{array}{l}\text { Construction de } \\
\text { ainsi que/valeur }\end{array}$ & Caractéristique/ & $\begin{array}{l}\mathrm{MF} \\
\mathrm{N}^{\circ} \text { exemple }\end{array}$ & $\begin{array}{l}\text { FM } \\
\text { Exemple-type }\end{array}$ \\
\hline Adjonction & & 56 & Pierre appuie la proposition ainsi que Jean \\
\hline \multirow[t]{2}{*}{ + subordonnée } & Corrélation & $1-2$ & $\begin{array}{l}\text { Ainsi que l'ébéniste agence ses pièces de bois, ainsi } \\
\text { l'écrivain agence ses mots }\end{array}$ \\
\hline & Intégration & $13-14-15$ & il a agi ainsi qu'il l'a toujours fait \\
\hline \multirow[t]{6}{*}{ Comparative } & Répétition du V & & $\begin{array}{l}\text { Ainsi que trop d'impôt tue l'impôt, trop d'Etat } \\
\text { tue l'Etat }\end{array}$ \\
\hline & Auxiliaire/proverbe & $6-7-8$ & il a agi ainsi qu'il l'a toujours fait \\
\hline & V lexical & & Il est ressorti ainsi qu'il était entré \\
\hline & V modal & 12 & $\begin{array}{l}\text { Ainsi que le voulait la coutume, il fit d'abord une } \\
\text { révérence à la déesse Soleil }\end{array}$ \\
\hline & V dire/perception & $10-11$ & $\begin{array}{l}\text { Ainsi que disent les braves gens, il bat la } \\
\text { campagne }\end{array}$ \\
\hline & Portée prédicative & & $\begin{array}{l}\text { Ainsi que son nom l'indique, Monsieur Pain est } \\
\text { boulanger }\end{array}$ \\
\hline \multirow[t]{2}{*}{ Temporelle } & $\begin{array}{l}\text { Portée } \\
\text { prédicationnelle }\end{array}$ & & $\begin{array}{l}\text { Ainsi que les amateurs de vin parlent en } \\
\text { Millésimes, les Anglais mesurent la valeur de leur } \\
\text { jeunesse universitaire aux performances des } \\
\text { équipages }\end{array}$ \\
\hline & Simultanéité & «Alors & \\
\hline
\end{tabular}




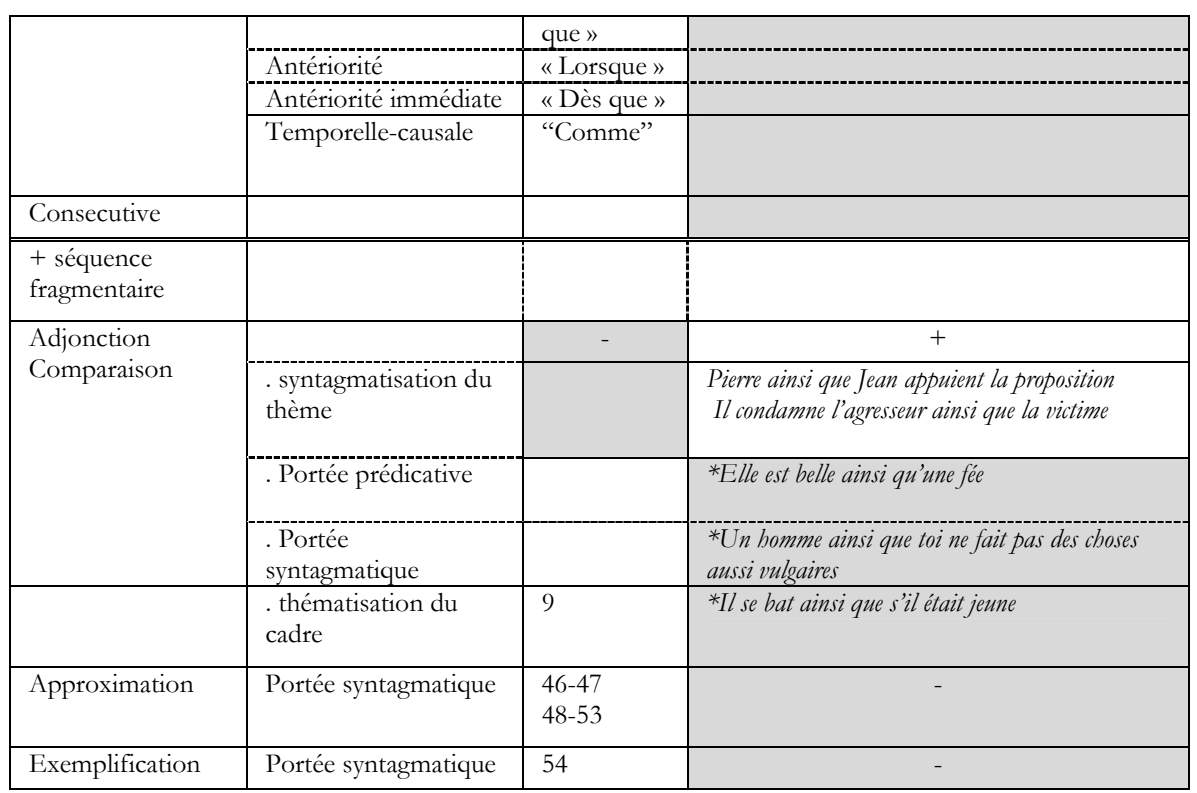

